\section{Dynamic thermal mechanical characterization of Epoclad negative photoresist for micro} mechanical structures

Kristof Wouters, Robert Puers.

KULeuven, ESAT-MICAS, Kasteelpark Arenberg 10, 3001 Leuven. e-mail: kristof.wouters@esat.kuleuven.be

Keywords: polymer MEMS, Epoclad, mechanical properties.

Abstract: Dynamic thermal mechanical properties of Epoclad negative photoresist have been studied. These experiments are part of the mechanical characterization of several photo definable polymers which can be used as a permanent, active and, or structural layer in a MEMS. This paper reports on the viscoelastic properties such as the loss modulus, the storage modulus, the glass transition temperature etc.

Text: The use of polymer micro mechanical structures enables the manufacture of cheaper MEMS. This is especially interesting for low end, consumer MEMS. Epoxy based polymers are a good candidate for use as a permanent, active or passive structural part of a micro electro mechanical device. Figure 1 gives for a sine ax a lithograhy the focus is chnology (GmbH) company [1].

Epoclad is very analog to SU-8. It can be patterned using UV lithography with various thicknesses, see Figure 2. It is also epoxy based, which makes it very stable in the mechanical domain but also in the chemical domain. The Epoclad resist is very difficult to remove, like the SU-8 resist. Epoclad, however, has superior adhesion properties with respect to SU-8 on various substrates.

Several mechanical properties of Epoclad, such as the Young's modulus, creep behavior, internal stress and the coefficient of thermal expansion, were already reported in previous publications [2, 3]. In this paper, the dynamic mechanical behavior at different temperatures is reported. According to the authors knowledge, no extensive dynamical responses of Epoclad have been published yet.

A major concern in using a polymer material as the moving part of a MEM is its creep behavior and the stiffness dependency on the frequency. As most polymers, the Epoclad is visco-elastic. This means that the material will be stiffer when deformed faster. It also means that additional losses will be introduced by the material when used in a resonator. A good characterization of this visco-elastic behavior is thus mandatory.

The dynamic thermal mechanical experiments were performed on a DMA Q 800 tool of the TA company. The measurements were done on freestanding films of Epoclad of $30 \mu \mathrm{m}$ to $60 \mu \mathrm{m}$ thick. Typically a sinusoidal stress or strain is applied to the sample at different temperatures and frequencies. From the obtained data the glass transition temperature (Tg) versus curing conditions, Figure 3 , the loss modulus, the storage modulus, Figure 4, the linear visco-elastic region (LVR), Figure 5, and frequency dependent stiffness's can be determined. From the LVR we can estimate until which strain the material can be regased a liner visco-lastic solid. The loss modulus will give us inforis be when a polym a the Youn be compared with the Young's modulus obtained via a standard tensile test. The complete experimental details and discussions will be given in the full paper.
References

1. H. Schroder, et. al., 2006 Waveguide and packaging technology for optical backplanes and hybrid electrical-optical circuit boards Proc of SPIE $\mathbf{6 1 2 4} 612407-14$.

2. K. Wouters, R. Puers, Young's modulus measurement and creep behavior of three different photodefinable epoxies for micro-fabrication, Proceedings EurosensorsXXII, Dresden, Germany, 7-10 September 2008, pp.835-838.

3. K. Wouters, R. Puers, Measurement of internal stress and coefficient of thermal expansion of epoclad negative photo resist, Proceedings of the MME'08, Aachen, 2008, pp.247-250

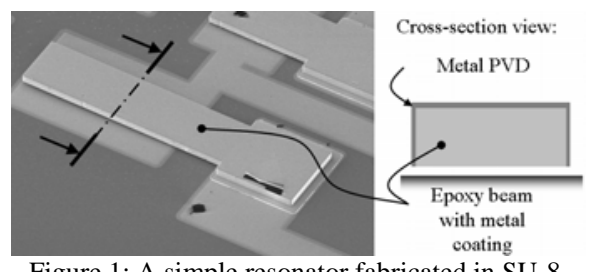

Figure 1: A simple resonator fabricated in SU-8.

The beam is $400 \mu \mathrm{m}$ long, $10 \mu \mathrm{m}$ high with a free

gap of $3 \mu \mathrm{m}$. The beam is coated with a metal using

sputtering to render the structure conductive.

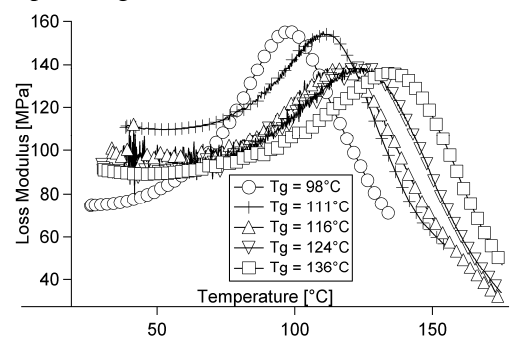

Figure 3: The recorded loss modulus's of samples which are cured differently. When increasing the curing, the Tg will become higher.

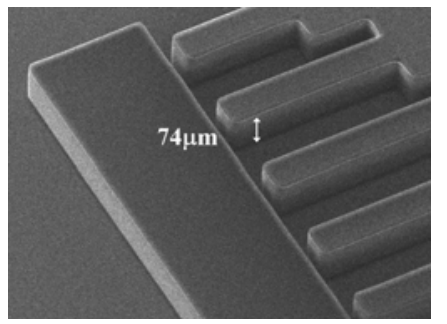

Figure 2: A thick patterned layer of Epoclad.

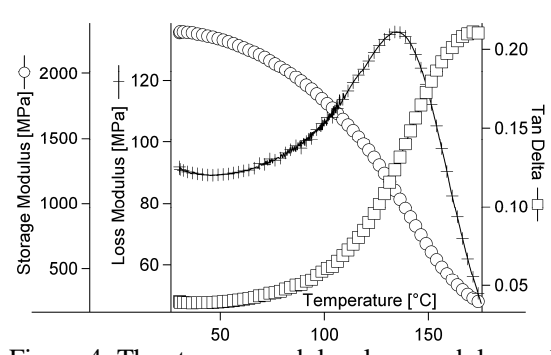

Figure 4: The storage modulus, loss modulus and angents of the phase shift versus the temperature at $1 \mathrm{~Hz}$.

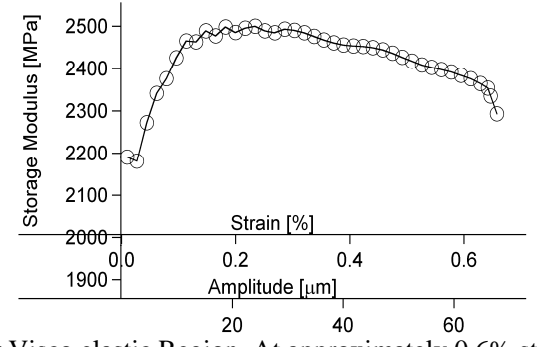

Figure 5: LVR, the Linear Visco elastic Region. At approximately 0.6\% strain the Epoclad can not be modeled as a linear visco elastic solid any more. 